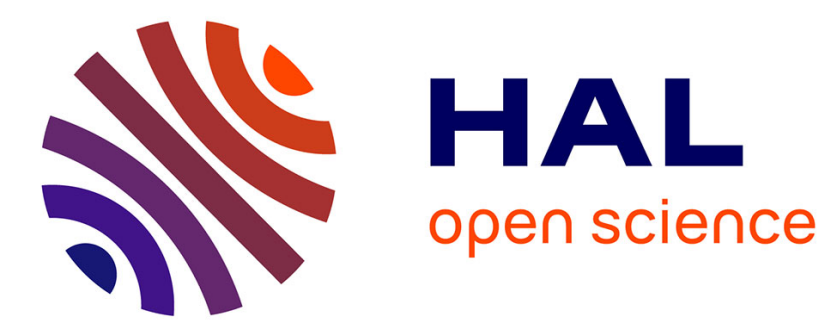

\title{
Spatial Issues in Modeling LoRaWAN Capacity
}

Andrzej Duda, Martin Heusse

\section{To cite this version:}

Andrzej Duda, Martin Heusse. Spatial Issues in Modeling LoRaWAN Capacity. Proceedings of the 22Nd International ACM Conference on Modeling, Analysis and Simulation of Wireless and Mobile Systems, Nov 2019, Miami Beach, United States. pp.191-198, 10.1145/3345768.3355932 • hal02378078

\section{HAL Id: hal-02378078 \\ https://hal.science/hal-02378078}

Submitted on 24 Nov 2019

HAL is a multi-disciplinary open access archive for the deposit and dissemination of scientific research documents, whether they are published or not. The documents may come from teaching and research institutions in France or abroad, or from public or private research centers.
L'archive ouverte pluridisciplinaire HAL, est destinée au dépôt et à la diffusion de documents scientifiques de niveau recherche, publiés ou non, émanant des établissements d'enseignement et de recherche français ou étrangers, des laboratoires publics ou privés. 


\section{Spatial Issues in Modeling LoRaWAN Capacity}

\author{
Andrzej Duda \\ andrzej.duda@imag.fr \\ Univ. Grenoble Alpes, CNRS, Grenoble INP, LIG \\ Grenoble, France
}

\begin{abstract}
All existing models for analyzing the performance of LoRaWAN assume a constant density of nodes within the gateway range. We claim that such a situation is highly unlikely for LoRaWAN cells whose range can attain several kilometers in real-world deployments. We thus propose to analyze the LoRa performance under a more realistic assumption: the density of nodes decreases with the inverse square of the distance to the gateway.

We use the LoRaWAN capacity model by Georgiou and Raza to find the Packet Delivery Ratio (PDR) for an inhomogeneous spatial distribution of devices around a gateway and obtain the number of devices that benefit from a given level of PDR. We analyze the LoRaWAN capacity in terms of PDR for various spatial configurations and Spreading Factor allocations.
\end{abstract}

\section{CCS CONCEPTS}

- Networks $\rightarrow$ Network performance modeling; Very longrange networks; Sensor networks.

\section{KEYWORDS}

LoRaWAN; Packet Delivery Ratio; capacity; modeling; inhomogeneous density

\section{ACM Reference Format:}

Andrzej Duda and Martin Heusse. 2019. Spatial Issues in Modeling LoRaWAN Capacity. In 22nd Int'l ACM Conference on Modeling, Analysis and Simulation of Wireless and Mobile Systems (MSWiM '19), November 25-29, 2019, Miami Beach, FL, USA. ACM, New York, NY, USA, 8 pages. https://doi.org/10.1145/3345768.3355932

\section{INTRODUCTION}

LoRa [1] is a recent example of a Low Power Wide Area Network (LPWAN) that can provide wireless connectivity to a large number of IoT devices over long distances. It defines a physical layer based on the Chirp Spread Spectrum (CSS) modulation [2] and a simple channel access method similar to ALOHA called LoRaWAN [3]. The LoRa CSS modulation results in good sensitivity enabling transmissions over long distances: a range of several kilometers outdoors and hundreds of meters indoors.

A LoRa end device can vary several transmission parameters: channel bandwidth (BW), transmission power (TP), coding rate

Permission to make digital or hard copies of all or part of this work for personal or classroom use is granted without fee provided that copies are not made or distributed for profit or commercial advantage and that copies bear this notice and the full citation on the first page. Copyrights for components of this work owned by others than ACM must be honored. Abstracting with credit is permitted. To copy otherwise, or republish, to post on servers or to redistribute to lists, requires prior specific permission and/or a fee. Request permissions from permissions@acm.org.

MSWiM '19, November 25-29, 2019, Miami Beach, FL, USA

(c) 2019 Association for Computing Machinery.

ACM ISBN 978-1-4503-6904-6/19/11 ..\$15.00

https://doi.org/10.1145/3345768.3355932

\author{
Martin Heusse \\ martin.heusse@imag.fr \\ Univ. Grenoble Alpes, CNRS, Grenoble INP, LIG \\ Grenoble, France
}

(CR), and spreading factor (SF). The achievable data rates depend on some of the parameters: a higher bit rate results from lower SF (at the cost of a shorter range), higher BW, and CR of $4 / 5$. The bit rates range from $293 \mathrm{~b} / \mathrm{s}$ to $11 \mathrm{~kb} / \mathrm{s}$, the low bit rate resulting in long transmission times: $2.466 \mathrm{~s}$ for sending $59 \mathrm{~B}$ at SF12.

In addition to the physical layer parameters, the LoRa performance in terms of the Packet Delivery Ratio (PDR) and scalability to a large number of devices strongly depend on the LoRaWAN access method similar to unslotted ALOHA: a device may wake up at any instant and start transmitting a packet without testing for on-going transmissions.

The previous analytical studies investigated LoRa performance for an increasing number of nodes around a gateway with an important assumption: the density of nodes within the gateway range is uniform [4-9]. However, such an assumption is not realistic for cells covering large areas of several square kilometers for two reasons.

First, measurement studies in cellular networks showed that spatial traffic distribution is highly non-uniform across different cells [10-14] with complex patterns that include hot spots with a high density and other less dense places. For instance, Lee et al. [11] demonstrated based on traffic measurements that the spatial distribution of the traffic density across different cells can be approximated by the log-normal or Weibull distributions depending on time and space. As cells in cellular networks can be smaller than LPWANs (target range of several kilometers), we expect that spatial traffic distribution in LoRa networks will also be highly non-uniform. We can also consider that the distribution of devices follows in fact the same pattern as we can usually observe for population and building densities in cities: apart from the saturated downtown, the density decreases with the distance from the centerit is much higher downtown than in suburbs. The deployment of gateways by LPWAN operators will probably also follow the same strategy as in cellular networks-place networks close to potential users and create hot spots near high density areas.

Second, there is a discouraging effect when placing a device far from the gateway in a LoRaWAN cell: the device needs to use large SF (e.g., SF11 or SF12) to get its transmissions through, which means long transmission times, so increased contention (more collisions) and higher energy consumption. For instance, a device using SF11 will roughly consume 10 times more energy for a transmission than when using SF7. Moreover, if we consider an equidistant distribution of spreading factors, which is the most popular spatial model adopted in the analyses with concentric annuli spaced at 1 $\mathrm{km}$ intervals, there are more devices with larger SF such as SF11 and SF12 than devices in the area of SF7. There are also no devices outside the zone of SF12, a kind of a disruptive irregularity difficult to observe in real world deployments.

We claim that uniform node density is highly unlikely for large cells so we propose to analyze LoRa performance under a more 
realistic assumption: the density of nodes decreases with the inverse square of the distance to the gateway. The inverse-square law is common in physics stating that a specified physical quantity or intensity is inversely proportional to the square of the distance from the source of that physical quantity. For instance, radio wave transmission in free space follows an inverse square law for power density. We consider that such a spatial model corresponds better to real-world LoRaWAN deployments than the models based on the constant density.

In this paper, we use the model by Georgiou and Raza [4] to find the Packet Delivery Ratio (PDR) for inhomogeneous spatial distributions of devices around a gateway and obtain the number of devices that benefit from a given level of PDR. Unlike Georgiou and Raza, we adopt a realistic assumption about generated traffic: nodes send packets at the smallest possible time interval determined by the largest duty cycle possible at SF12. Under this assumption, devices generate packets in a way defined by a sensing application unlike the model by Georgiou and Raza that assumes $1 \%$ duty cycle of all devices regardless of SF, which means that a device that changes from SF8 to SF7 starts sending packets twice more often. We also modify the model expression for traffic intensity so it correctly reflects the unslotted ALOHA behavior (instead if slotted ALOHA).

In the rest of the paper, we describe the basics of LoRa networks (Section 2) and present the PDR model (Section 3). In Section 4, we analyze LoRaWAN capacity in terms of PDR for different spatial configurations. Finally, we discuss related work (Section 5) and draw some conclusions (Section 6).

\section{LORAWAN BASICS}

We briefly recall the basic characteristics of LoRaWAN.

Devices can control the physical layer of LoRaWAN through the following parameters [15]:

- Bandwidth (BW): it is the range of transmission frequencies. We can configure the bandwidth between $7.8 \mathrm{kHz}$ and 500 $\mathrm{kHz}$. A larger bandwidth allows for a higher data rate, but results in lower sensitivity.

- Spreading Factor (SF) characterizes the number of bits carried by a chirp: $\mathrm{SF}$ bits are mapped to one of $N=2^{\mathrm{SF}}$ possible frequency shifts in a chirp. SF varies between 6 (7 in practice) and 12 , with SF12 resulting in the best sensitivity and range, at the cost of achieving the lowest data rate and worst energy consumption. Decreasing the SF by 1 unit roughly doubles the transmission rate and divides by 2 the transmission $\mathrm{du}-$ ration as well as energy consumption.

- Coding Rate (CR): it corresponds to the rate of Forward Error Correction (FEC) applied to improve packet error rate in presence of noise and interference. A lower coding rate results in better robustness, but increases the transmission time and energy consumption. The possible values are: $4 / 5$, $4 / 6,4 / 7$, and $4 / 8$.

- Transmitted Power (TP): LoRaWAN defines the following values of TP for the EU 863-870 MHz band: $2 \mathrm{dBm}, 5 \mathrm{dBm}$, $8 \mathrm{dBm}, 11 \mathrm{dBm}$, and $14 \mathrm{dBm}$.

Table 1 presents $\mathrm{SF} j$, data rate $\mathrm{DR} j$, Signal-to-Noise Ratio (SNR) limit, airtime $\tau_{j}$, and $s_{\max }$, the maximum payload size. $\tau_{j}$ denotes
Table 1: LoRa parameters for BW of $125 \mathrm{kHz}$.

\begin{tabular}{|c|c|c|c|c|}
\hline $\mathrm{SF} j$ & $\begin{array}{r}\text { SNR limit } \\
q_{j}\end{array}$ & $\begin{array}{r}\text { Airtime } \\
\tau_{j}\end{array}$ & $\begin{array}{r}\text { Bit rate } \\
\text { DRj }\end{array}$ & $s_{\max }$ \\
\hline 7 & $-7.5 \mathrm{~dB}$ & $102.7 \mathrm{~ms}$ & DR5: $5469 \mathrm{~b} / \mathrm{s}$ & $230 \mathrm{~B}$ \\
\hline 8 & $-10 \mathrm{~dB}$ & $184.8 \mathrm{~ms}$ & DR4: $3125 \mathrm{~b} / \mathrm{s}$ & $230 \mathrm{~B}$ \\
\hline 9 & $-12.5 \mathrm{~dB}$ & $328.7 \mathrm{~ms}$ & DR3: $1758 \mathrm{~b} / \mathrm{s}$ & $123 \mathrm{~B}$ \\
\hline 10 & $-15 \mathrm{~dB}$ & $616.5 \mathrm{~ms}$ & $977 \mathrm{~b} / \mathrm{s}$ & $59 \mathrm{~B}$ \\
\hline 11 & $-17.5 \mathrm{~dB}$ & $1315 \mathrm{~ms}$ & $537 \mathrm{~b} / \mathrm{s}$ & $59 \mathrm{~B}$ \\
\hline 12 & $-20 \mathrm{~dB}$ & 2466 & $293 \mathrm{~b} / \mathrm{s}$ & $59 \mathrm{~B}$ \\
\hline
\end{tabular}

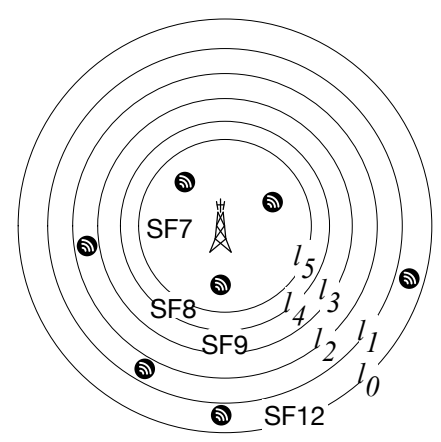

Figure 1: Annuli of SF configurations around a gateway

the transmission duration of the maximum size frame at data rate DRj.

LoRaWAN defines an access method similar to ALOHA: a device wakes up at any instant and sends a packet right away. It then wakes up after a delay to receive a downlink frame, if the transmitted frame was of the Confirmed Data type. Collisions occur when two devices transmit packets at the same time. However, unlike in the pure ALOHA scheme, a receiver can correctly receive a frame in presence of interfering signals, because the LoRa physical layer is robust enough to resist significant interference. The capture effect has important impact on LoRa performance. Taking into account the capture effect, Haxhibeqiri et al. [16] showed that when the number devices increases to 1000 per gateway, the packet loss rate only increases to $32 \%$, which is low compared to $90 \%$ in pure ALOHA for the same load.

In the following sections, we assume LoRa modulations with $125 \mathrm{kHz}$ bandwidth and spreading factors $\mathrm{SF}\{12-j\}$ that result in data rates $\mathrm{DR} j$, with $j=0,1, \ldots 5$ (see Table 1 ).

\section{MODEL FOR SUCCESSFUL PACKET DELIVERY}

We consider a LoRaWAN cell in which devices choose SF (so the data rate) based on the distance to the gateway, which corresponds to the annuli view presented in Figure $1 . l_{j}$ denotes the distance to the farthest device that uses $\mathrm{SF}\{12-j\}$ so its data rate is DRj. $l_{0}$ is the maximum transmission range.

We assume that devices are located at random in the annulus at $l_{j}$ according to a Poisson Point Process (PPP) with intensity $\rho_{j}$. Inside the annulus, the spatial density is constant, so the number 
Table 2: Equidistant SF boundaries $[\mathbf{k m}], S_{j} / \pi\left[\mathbf{k m}^{2}\right]$ : value proportional to the number of devices (constant node density $\rho$ in all annuli).

\begin{tabular}{crrrrrr} 
& SF7 & SF8 & SF9 & SF10 & SF11 & SF12 \\
& $l_{5}$ & $l_{4}$ & $l_{3}$ & $l_{2}$ & $l_{1}$ & $l_{0}$ \\
\hline$l_{j}[\mathrm{~km}]$ & 1 & 2 & 3 & 4 & 5 & 6 \\
$S_{j} / \pi\left[\mathrm{km}^{2}\right]$ & 1 & 3 & 5 & 7 & 9 & 11 \\
\hline
\end{tabular}

Table 3: Notation

\begin{tabular}{lc} 
Traffic generation intensity & $\lambda_{t}$ \\
Frame transmission duration at data rate DRj & $\tau_{j}$ \\
Distance of farthest node using DRj & $l_{j}$ \\
Surface of annulus $j$ & $S_{j}$ \\
Spatial density of nodes in annulus $j$ & $\rho_{j}$ \\
Total number of nodes & $n$ \\
Average number of nodes in annulus $j$ & $n_{j}$ \\
Traffic occupancy (in Erlang) at DRj & $v_{j}$ \\
Average channel gain at distance $d$ & $g(d)$ \\
SNR threshold for DRj & $q_{j}$ \\
Transmission power, in-band noise power & $P, N$ \\
Success probability, due to attenuation, fading & $H$ \\
Success probability, due to collisions & $Q_{1}$ \\
\hline
\end{tabular}

of nodes using $\operatorname{SF}\{12-j\}$ and data rate $\mathrm{DR} j$ is proportional to $\rho_{j}$ and the surface of the annulus between $l_{j+1}$ and $l_{j}$.

Table 2 presents the values of $l_{j}$ for the equidistant SF configuration with maximum range $l_{0}$ of $6 \mathrm{~km}$ and a homogeneous density (Mahmood et al. considered such boundaries [7] and Georgiou and Raza provided numerical examples for the double maximum range: $l_{0}$ of $12 \mathrm{~km} \mathrm{[4]).} \mathrm{The} \mathrm{number} \mathrm{of} \mathrm{devices} \mathrm{in} \mathrm{a} \mathrm{disk} \mathrm{or} \mathrm{annulus} \mathrm{is}$ proportional to surface $S_{j}$ so it increases with the distance. Note that energy consumption of a node in annulus $j$ is proportional to airtime $\tau_{j}$, which attains large values for high SF.

We give below the details of the model [4] to compute PDR based on the notation in Table 3.

Provided that there is no collision, a frame transmission succeeds as long as the SNR at the receiver for this transmission is above $q_{j}$, the minimum SNR for the corresponding spreading factor [17]. We consider a Rayleigh channel, so that the received signal power is affected by a multiplicative random variable with an exponential distribution of unit mean (and standard deviation). Recent measurements confirm the validity of the hypothesis for LoRa transmissions [18]. Thus, the signal power depends on the distance and the Rayleigh fading gain, whereas the noise power is the constant thermal noise for a $125 \mathrm{kHz}$-wide band: $N=-123 \mathrm{dBm}$. We consider the maximum transmission power of $P=14 \mathrm{dBm}$.

Thus, the probability of successful transmission at distance $l_{j}$ with data rate $\mathrm{DR} j$ is [4]:

$$
H\left(l_{j}\right)=\exp \left(-\frac{N q_{j}}{P g\left(l_{j}\right)}\right),
$$

where $g\left(l_{j}\right)$ is the average channel gain at distance $l_{j}$. We use the Okumura-Hata model for path loss attenuation.
We use an approximate expression for the success probability in presence of concurrent traffic [4] reflecting the behavior of unslotted ALOHA with capture:

$$
Q_{1}\left(l_{j}, v_{j}\right)=\frac{2 \exp \left(-2 v_{j}\right) l_{j}^{\eta}(\eta+2) S_{j}}{\pi 2 v_{j} l_{j}^{\eta+2}+l_{j}^{\eta}\left(2(\eta+2) S_{j}-2 \pi v_{j} l_{j}^{2}\right)},
$$

where $\eta$ is the path loss exponent (we assume $\eta=4$ in the numerical examples below, which gives relative attenuation values that closely match those of the propagation model). Note that we double the traffic intensity in the expression by Georgiou and Raza to reflect correctly the behavior of unslotted ALOHA.

Finally, PDR of nodes in annulus $j$ is the following:

$$
\operatorname{PDR}\left(l_{j}, v_{j}\right)=H\left(l_{j}\right) \times Q_{1}\left(l_{j}, v_{j}\right)
$$

To use these expressions, we need to define traffic intensity $v_{j}$. We consider that nodes generate traffic according to a Poisson process of intensity $\lambda_{t}$. For SF $=12-j$ and $n_{j}$, the number of contending nodes in annulus $j$, traffic occupancy is

$$
v_{j}=n_{j} \tau_{j} \lambda_{t}
$$

in Erlang and the number of nodes in annulus $j$ is:

$$
n_{j}=\frac{S_{j} \rho_{j} n}{\sum_{j} S_{j} \rho_{j}}
$$

We set $\lambda_{t}$ to the traffic intensity of nodes operating at DR0 and SF12 at their maximal duty cycle and using $59 \mathrm{~B}$ packets, the maximum size at this rate. The duty cycle depends on the frequency band: LoRa devices have to limit their occupation of each frequency band to $1 \%$ of time with 3 to 5 frequency channels in each band in Europe. The airtime of maximum size packets at DR0 corresponds to $2.47 \mathrm{~s}$ so they can be sent every $747 \mathrm{~s}$ to achieve $0.33 \%$ duty cycle per frequency channel. Thus,

$$
\lambda_{t}=\frac{1}{747 \mathrm{~s}} .
$$

\section{PROBABILITY OF SUCCESSFUL PACKET DELIVERY FOR DIFFERENT SPATIAL CONFIGURATIONS}

In this section, we analyze PDR for different spatial configurations.

\subsection{SF Allocation Strategies}

There are several ways of allocating SF to nodes, which means finding the annuli boundaries $l_{j}$ (we restrict our analysis to nonoverlapping allocations in which all nodes in a given annulus use the same value of SF) $[5,7,19]$ :

(1) Equidistant SF allocation with $l_{j+1}-l_{j}=l_{0} / 6$ (also called equal-interval-based [19]).

(2) Equal-area-based SF allocation with $l_{j}=l_{0} \sqrt{j / 6}$ [19].

(3) SNR-based SF allocation with $l_{j}=\{d: H(d) \geq \theta\}$ (also called path loss based [7]).

(4) PDR-based SF allocation with $l_{j}=\left\{d: H(d) \times Q_{1}(d) \geq \theta\right\}$.

Mahmood et al. showed that equidistant and SNR-based SF allocations performed the best, nevertheless, they did not consider the PDR-based allocation [7]. Lim and Han analyzed the PDR-based 


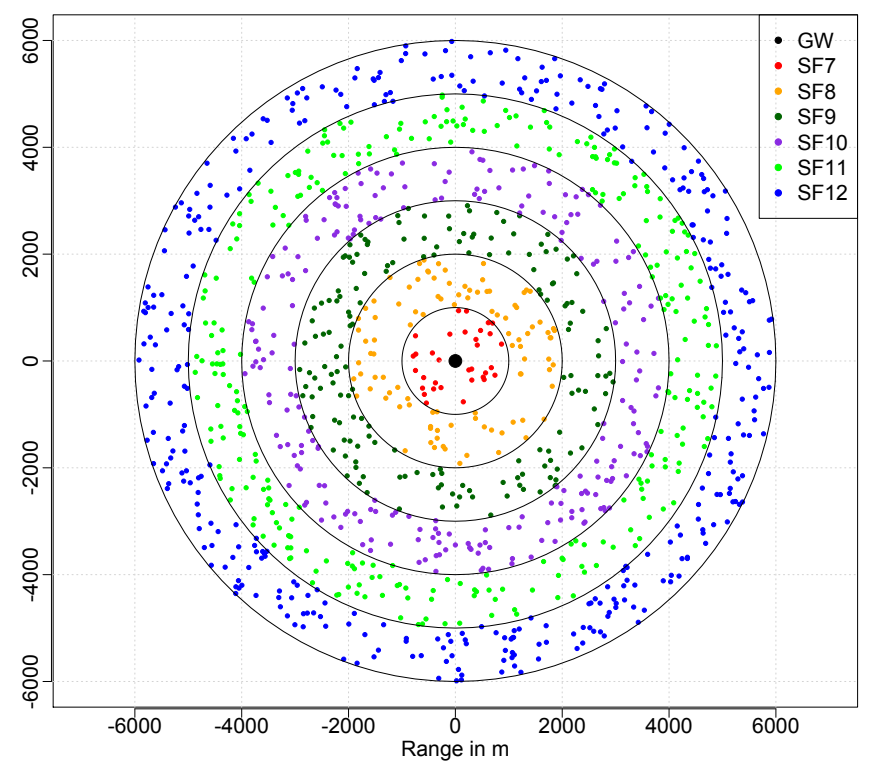

(a) Homogeneous density

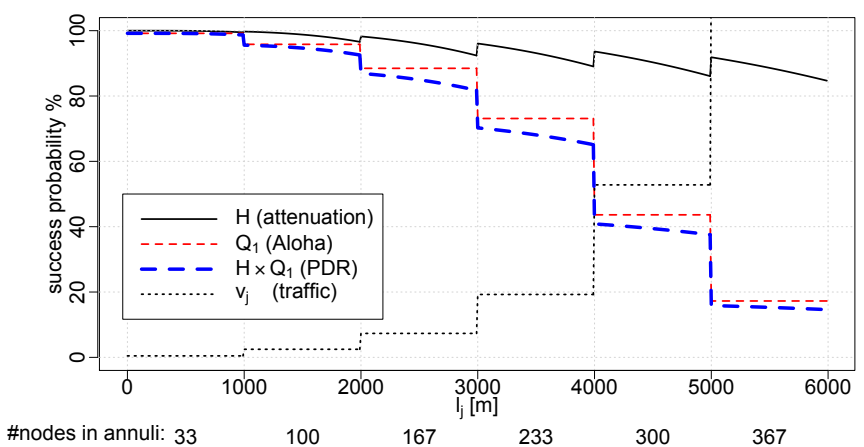

(c) PDR for homogeneous density. 300 nodes with PDR $>80 \%$

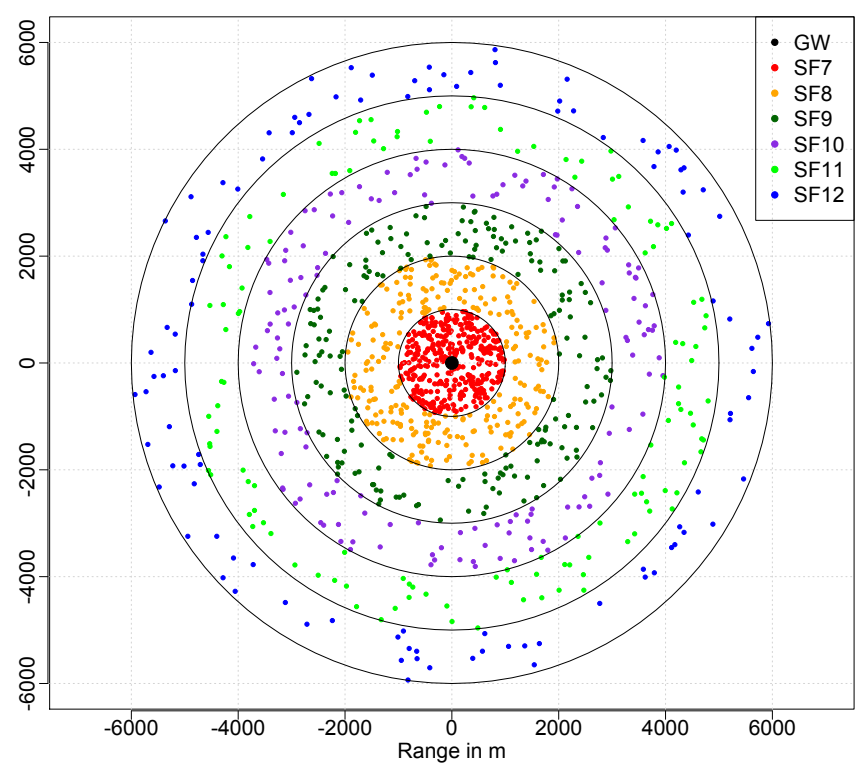

(b) Inverse squared density

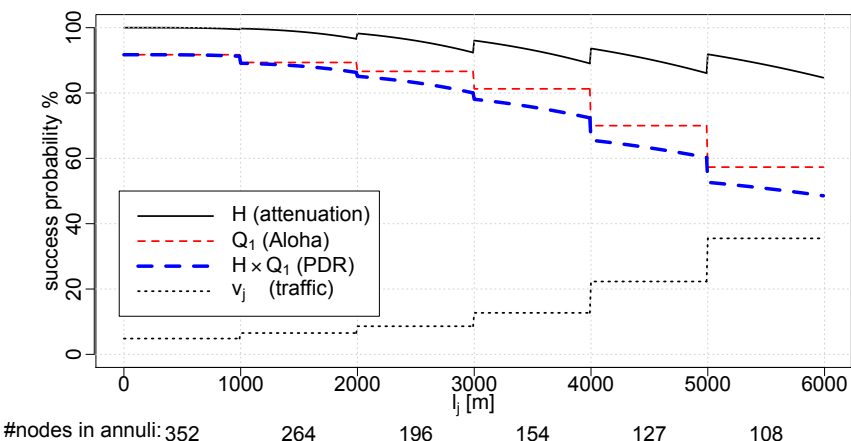

(d) PDR for inverse squared density. 809 nodes with PDR $>80 \%$

Figure 2: Comparison of spatial models, $n=1200$.

SF allocation and showed that it performs the best with the equalarea-based SF allocation ranked second [19]. However, they did not take into account the capture effect important to evaluate the probability of success reception under the LoRaWAN access method. The analyses assumed a constant node density within the range.

Note that the complexity of computing SF allocations increases with the order of allocations presented above: equidistant and equalarea-based allocations only depend on the distance, the SNR-based allocation requires solving Eq. 1 numerically, and PDR-based allocations lead to a nonlinear optimization problem.

The SNR-based allocation resulting from solving Eq. 1 numerically for each $j$ gives the values of $l_{j}$ presented in Table 4 for three values of threshold $\theta: 90 \%, 95 \%$, and $99 \%$. We can observe that increasing the threshold results in smaller cells.
The SNR-based and PDR-based allocations can be implemented in a similar way to the Adaptive Data Rate (ADR) algorithm defined in LoRaWAN. In ADR, the gateway estimates the average SNR level for the last 20 packets of a node. It then chooses SF and TP suitable for the given level of SNR, while keeping a $5 \mathrm{~dB}$ margin, and sends the parameters in the LinkADRReq frame to the node. In a similar way, the gateway can estimate PDR and choose the right parameters for the node. Nevertheless, such allocations require sending downlink messages, whereas the gateways have very limited transmission capacity.

\subsection{Inhomogeneous Node Density}

We have already discussed the reasons for which assuming constant node density $\rho$ for all annuli is not realistic. Moreover, the allocation of SF in LoRaWAN strongly impacts energy consumption so that far devices that need to use high values of SF will consume much 
Table 4: SNR-based SF boundaries [ $\mathbf{k m}], H\left(l_{j}\right)$ is the success probability due to attenuation, fading, thermal noise. $S_{j} / \pi$ $\left[\mathrm{km}^{2}\right]$ : value proportional to the number of devices. Node density in an annulus based on the inverse-square law so $S_{j} / \pi \times \rho\left(l_{j}\right)$ is proportional to the number of devices.

\begin{tabular}{crrrrrr} 
& SF7 & SF8 & SF9 & SF10 & SF11 & SF12 \\
$l_{5}$ & $l_{4}$ & $l_{3}$ & $l_{2}$ & $l_{1}$ & $l_{0}$ \\
\hline \hline$l_{j}: H\left(l_{j}\right) \geq 90 \%$ & 2.23 & 2.68 & 3.23 & 3.89 & 4.54 & 5.30 \\
$S_{j} / \pi\left[\mathrm{km}^{2}\right]$ & 4.96 & 2.23 & 3.24 & 4.69 & 5.49 & 7.47 \\
$\rho_{j}$ & 1 & 0.69 & 0.48 & 0.33 & 0.24 & 0.18 \\
$S_{j} / \pi \times \rho\left(l_{j}\right)$ & 4.96 & 1.54 & 1.54 & 1.54 & 1.32 & 1.32 \\
\hline$l_{j}: H\left(l_{j}\right) \geq 95 \%$ & 1.84 & 2.21 & 2.66 & 3.20 & 3.74 & 4.37 \\
$S_{j} / \pi\left[\mathrm{km}^{2}\right]$ & 3.38 & 1.50 & 2.19 & 3.17 & 3.74 & 5.1 \\
$\rho_{j}$ & 1 & 0.69 & 0.48 & 0.33 & 0.24 & 0.18 \\
$S_{j} / \pi \times \rho\left(l_{j}\right)$ & 3.38 & 1.03 & 1.05 & 1.05 & 0.9 & 0.9 \\
\hline$l_{j}: H\left(l_{j}\right) \geq 99 \%$ & 1.18 & 1.43 & 1.72 & 2.07 & 2.41 & 2.82 \\
$S_{j} / \pi\left[\mathrm{km}^{2}\right]$ & 1.40 & 0.63 & 0.91 & 1.33 & 1.55 & 2.11 \\
$\rho_{j}$ & 1 & 0.69 & 0.48 & 0.33 & 0.24 & 0.18 \\
$S_{j} / \pi \times \rho\left(l_{j}\right)$ & 1.40 & 0.44 & 0.44 & 0.44 & 0.37 & 0.37 \\
\hline
\end{tabular}

more energy than devices with low SF, which will discourage the placement of nodes far from the gateway. There is also another adverse effect of using high values of SF: increased transmission times lead to more contention and collisions, thus affecting the probability of successful packet reception.

To take into account these considerations, we adopt a more realistic model for the spatial distribution of nodes based on the inverse-square law for node density:

$$
\frac{\rho_{j}}{\rho_{j-1}}=\frac{l_{j-1}^{2}}{l_{j}^{2}}
$$

Table 4 also presents node density $\rho_{j}$ for each annulus based on this relation and the value proportional to the number of devices. To see the effect of the decreasing node density, we can observe that such a distribution favors the annuli close to the gateway with a higher number of devices using SF7 and results in less devices with SF 11 and SF12.

Figure 2 compares the spatial models-Figures $2 \mathrm{a}$ and $2 \mathrm{~b}$ visualize the distribution of nodes for homogeneous and inverse squared density ( $n=1200$ nodes generated with a Monte Carlo method, randomly placed in equidistant annuli). We can observe that the number of nodes in the SF12 annulus is much lower for inverse squared density: 108 vs. 367 (see Figures 2c and 2d).

\subsection{LoRaWAN capacity for different spatial configurations}

In this section, we present figures with PDR computed according to the model in Section 3. The total number of nodes $(1200,1700$, and 2100) for the figures was chosen so that they give the maximal number of nodes that benefit from PDR $>80 \%$ for the respective SNR thresholds (we discuss this aspect at the end of this Section).

4.3.1 Equidistant SF frontiers. Figures 2a and 2c present the spatial distribution of nodes and PDR in equidistant SF annuli for a

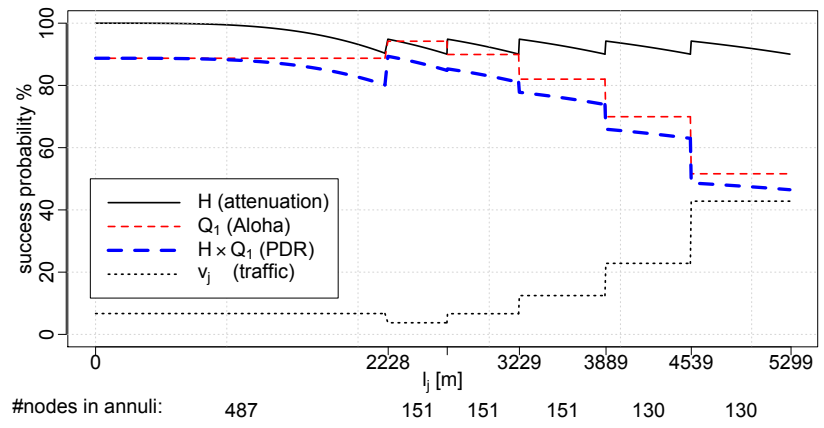

Figure 3: Inverse squared density and SNR SF annuli for $H\left(l_{j}\right) \geq 90 \%, n=1200.787$ nodes with PDR $>80 \%$.

homogeneous density and $n=1200$ nodes. The existing studies use this distribution and equidistance frontiers of SF allocation to analyze the LoRa capacity. Figure 2c shows PDR and its components: channel attenuation $H$ and $Q_{1}$, the success probability under ALOHA with capture. We can observe that $Q_{1}$ goes down at each frontier because of increased traffic $v_{j}$ that comes from an increased number of nodes. As the annuli surface increases with the distance, the homogeneous node density results in the number of nodes in annuli growing with the distance and attaining 367 for SF12 with only 33 nodes using SF7. The estimated number of nodes that benefit from PDR $>80 \%$ is 300 . The spatial model assumes that there are no nodes outside the last annuli.

Figures $2 \mathrm{~b}$ and $2 \mathrm{~d}$ present the distribution of nodes and PDR when the density of nodes is inversely proportional to the square of distance. PDR goes down with the distance much slowly than in Figure 2c because there are less nodes in higher SF annuli: 352 nodes using SF7 and 108 for SF12. The estimated number of nodes that benefit from PDR $>80 \%$ is 809 . We can observe that the number of nodes in each annulus decreases with the distance.

This basic example shows the importance of the spatial model for evaluating LoRaWAN capacity: just changing the spatial distribution of nodes raises the number of nodes with good PDR from 300 to 809 . So, the choice of the spatial model may result in misleading results on LoRaWAN capacity and scalability.

4.3.2 SNR-based SF frontiers. Figure 3 presents PDR in SNR-based SF allocation for the inverse squared density and $n=1200$ nodes. For $H\left(l_{j}\right) \geq 90 \%$ threshold, the range of the cell is relatively large with $5.3 \mathrm{~km}$. 787 nodes benefit from PDR $>80 \%$ out of 1200 .

Figure 4 and Figure 5 present the same data for $H\left(l_{j}\right) \geq 95 \%$ and $H\left(l_{j}\right) \geq 99 \%$ thresholds and the total number of nodes $n=1700$ and $n=2100$, respectively. The increased value of the threshold results in smaller cells $\left(2.82 \mathrm{~km}\right.$ for $\left.H\left(l_{j}\right) \geq 99 \%\right)$ because of the dependance of funtion $H$ on the distance. The number of nodes that benefit from PDR $>80 \%$ is 1115 and 1377, respectively.

We can observe that the assumption of the inhomogeneous density results in an interesting effect: smaller cells can provide good PDR for an increased number of nodes. It evokes "cell breathing" in cellular networks in which heavily loaded cells decrease in size. 


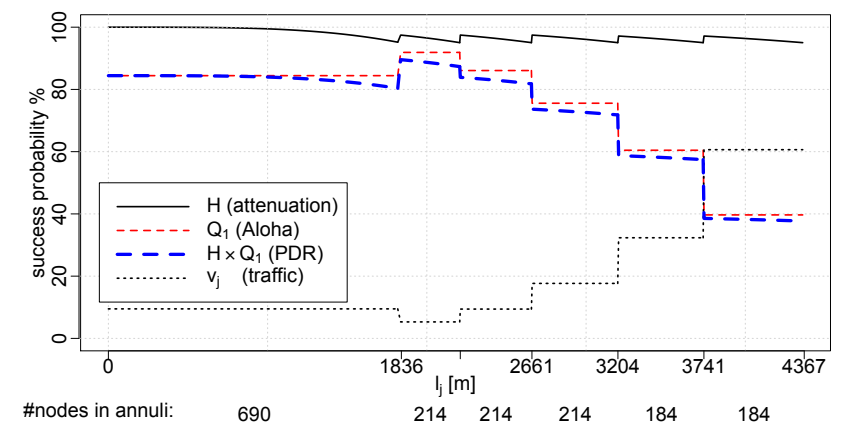

Figure 4: Inverse squared density and SNR SF annuli for $H\left(l_{j}\right) \geq 95 \%, n=1700.1115$ nodes with PDR $>80 \%$.

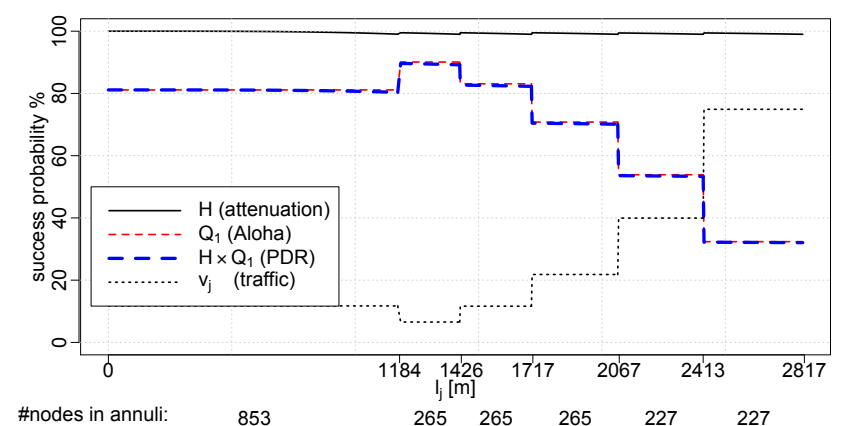

Figure 5: Inverse squared density and SNR SF annuli for $H\left(l_{j}\right) \geq 99 \%, n=2100.1377$ nodes with PDR $>80 \%$.

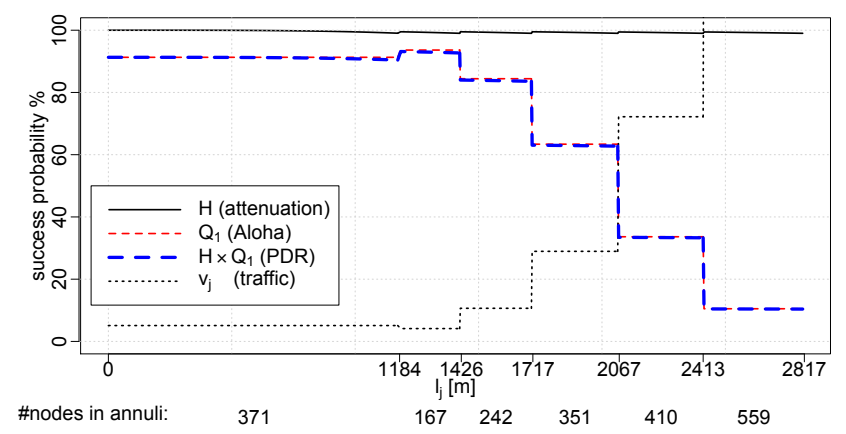

Figure 6: Homogeneous density and SNR SF annuli for $H\left(l_{j}\right) \geq 99 \%, n=2100.776$ nodes with PDR $>80 \%$.

Note that the nodes benefiting from PDR $>80 \%$, use low SF values: SF7, SF8, and SF9, which also means that their energy consumption stays low.

Figure 6 presents the results for a homogeneous density to compare with Figure 5: there are 776 nodes with PDR $>80 \%$.

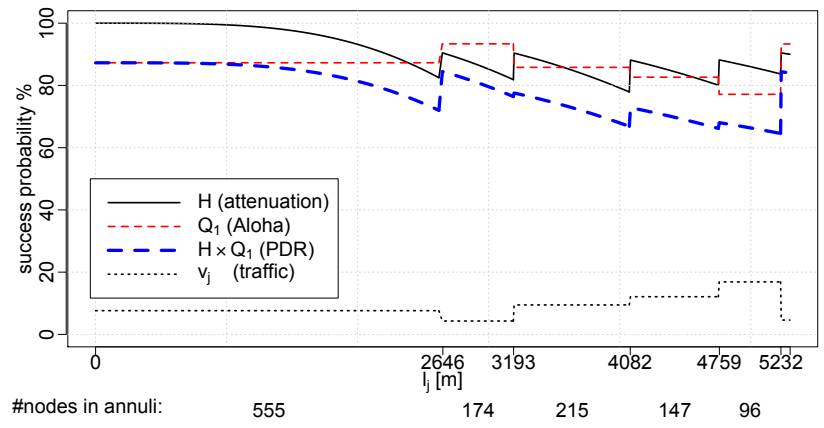

Figure 7: Inverse squared density and PDR SF annuli for $H\left(l_{j}\right) \geq 90 \%, n=1200.460$ nodes with PDR $>80 \%$.

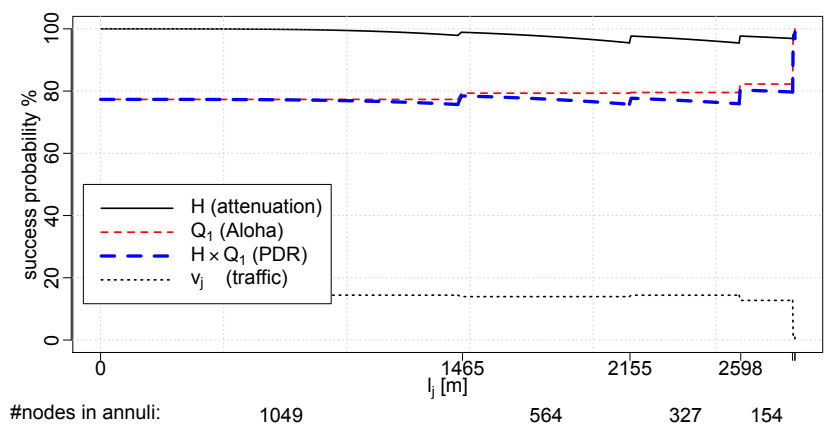

Figure 8: Inverse squared density and PDR SF annuli for $H\left(l_{j}\right) \geq 99 \%, n=2100$. No nodes with PDR $>80 \%$.

4.3.3 PDR-based SF frontiers. For PDR-based SF allocations, we use the Nelder-Mead simplex [20] to find

$$
\underset{l_{0} \ldots l_{5}}{\arg \max } \min \left(H \times Q_{1}\right) .
$$

The optimization starts with the maximal range $l_{0}$ that we set to the thresholds for two extreme SNR-based allocations: $5.30 \mathrm{~km}$ for $90 \%$ and $2.82 \mathrm{~km}$ for $99 \%$ (we skip the intermediate threshold of $95 \%$ ), and looks for more uniform distribution of PDR values across $\mathrm{SF}$ annuli with the maxmin objective. We still assume the density of nodes inversely proportional to the square of distance.

Figure 7 presents PDR in the first case of a large cell: $l_{0}=5.30 \mathrm{~km}$ for $90 \%$ for the same total number of nodes as in Figure 3. Compared to Figure 3, the minimum value of PDR is higher (PDR $>60 \%$ ), however, there are less nodes that benefit from good PDR $>80 \%$ (460 vs. 787).

Similarly, Figure 8 shows PDR for the small cell: $2.82 \mathrm{~km}$ for 99\%. When comparing with Figure 5, we can see a similar effectthe minimal value of PDR is high (almost reaching 80\%), but still the number of nodes that benefit from good PDR $>80 \%$ is low, which shows that the call has attained its capacity. If we lower the total number of nodes in the network to 1500, the maxmin PDR allocation gives very good results: Figure 9 shows that all nodes achieve PDR $>80 \%$. 


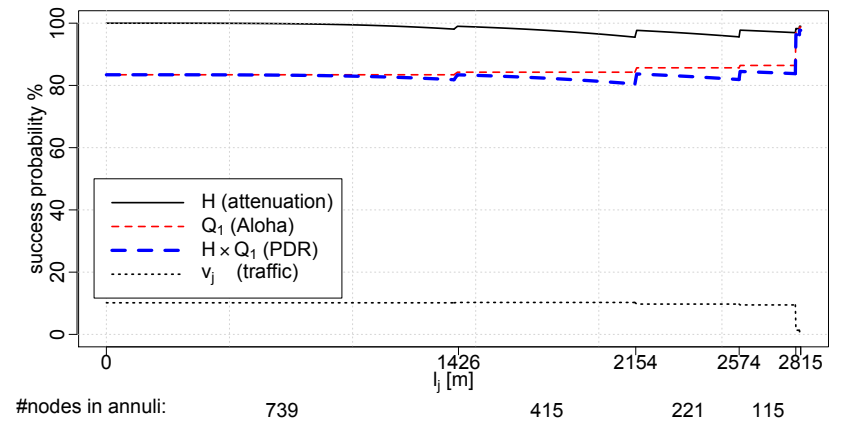

Figure 9: Inverse squared density and PDR SF annuli for $H\left(l_{j}\right) \geq 99 \%, n=1500$. All 1500 nodes with PDR $>80 \%$.

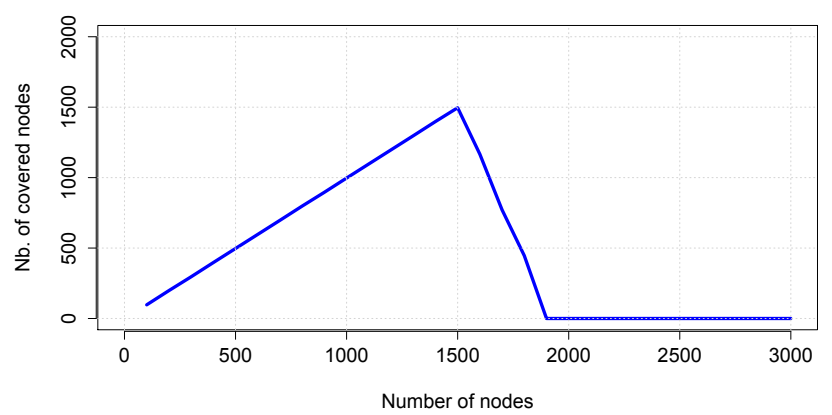

Figure 10: Number of nodes with PDR $>80 \%$ in function of the total number of nodes in the network. Optimal allocation of annuli frontiers $l_{j}$ as in Figure 9.

Note that the allocation exhibits strong asymmetry-it favors lower SF over high SF: there are 739 nodes in the SF7 annuli compared to only 8 nodes with SF12. As $H$ is as high as $99 \%$, the most important factor for PDR is $Q_{1}$, which depends on the traffic load almost constant for all annuli with low SF (see Figure 9) in this allocation. Such an allocation has also an advantage of low overall energy consumption as only a few nodes use SF11 and SF12, expensive in terms of energy.

4.3.4 Scalability. Figure 10 shows the number of nodes that benefit from good PDR $>80 \%$ in function of the total number of nodes in the network for the small cell of the $2.82 \mathrm{~km}$ range. We fix the allocation of annuli frontiers $l_{j}$ to the optimal allocation presented in Figure 9 and we vary the total number of nodes. At the beginning, the number of nodes with PDR $>80 \%$ increases and achieves the maximum for $n=1500$. Then, the number of nodes with PDR $>80 \%$ decreases because PDR begins to drop below $80 \%$, which means that the network has attained its capacity.

A question remains: how does the SNR allocation perform compared to the PDR based one? Figure 11 shows the corresponding data for the SNR allocation. It achieves a lower maximum number of nodes with PDR $>80 \%$ (1377 nodes for $n=2100$, see also Figure 5), but it can handle a slightly larger total number of nodes.

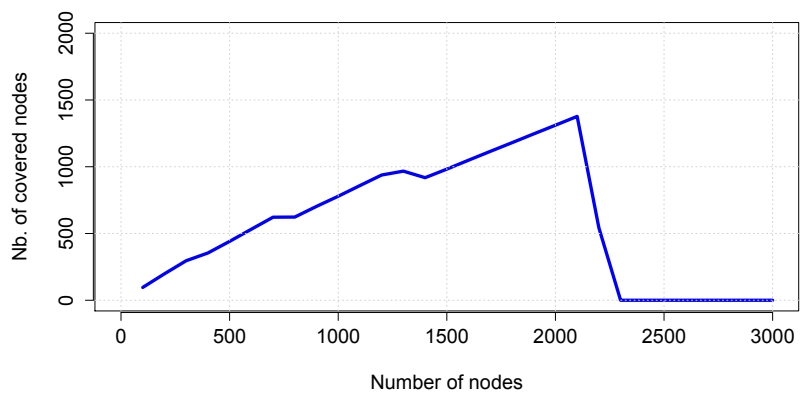

Figure 11: Number of nodes with PDR $>80 \%$ in function of the total number of nodes in the network. SNR allocation of annuli frontiers $l_{j}$.

\section{RELATED WORK}

We briefly review previous work on modeling LoRa capacity and inhomogeneous spatial models.

\subsection{LoRa capacity models}

Georgiou and Raza [4] provided a stochastic geometry framework for modeling the performance of a single gateway LoRa network. They showed that the coverage probability drops exponentially as the number of contending devices grows. Their model assumes that the airtime is filled up-nodes use the shortest interval between packet transmissions allowed at given SF, which means that switching to lower SF results in generating twice as much traffic. Moreover, the model of the access method corresponds to slotted ALOHA. In this paper, we have used the model with the modifications concerning the intensity of packet generation and the expression for the success probability reflecting the behavior of unslotted ALOHA.

Mahmood et al. [7] proposed an analytical model of a single-cell LoRa system that takes into account interference among transmissions over the same SF (co-SF) as well as different SFs (inter-SF). They derived the signal-to-interference ratio (SIR) distributions for several interference conditions. Due to imperfect orthogonality, inter-SF interference exposes the network for additional 15\% coverage loss for a small number of concurrently transmitting enddevices.

Li et al. [5] analyzed interference in the time-frequency domain using a stochastic geometry model assuming transmissions as patterns on a two-dimensional plane to quantify the capture effect. They use the model to analyze LoRaWAN by characterizing the outage probability and throughput.

Based on a simple model for collisions and capture effect, Caillouet et al. [9] introduced a theoretical framework for maximizing the LoRaWAN capacity in terms of the number of end nodes.

All the presented models assumed a homogeneous node density around a gateway.

\subsection{Inhomogeneous spatial models}

Gotzner and Rathgeber [10] challenged the homogeneous assumption in spectrum frequency analysis and proposed to model the 
spatial inhomogeneity of real cellular traffic with log-normal distributions.

Lee et al. [11] observed that modeling and simulation of a cellular network typically assume the target area divided into regular hexagonal cells and a uniform distribution of mobile devices scattered in each cell. In reality, the spatial traffic distribution is highly non-uniform across different cells, which requires adequate spatial traffic models. They reported on traffic measurements collected from commercial cellular networks and demonstrated that the spatial distribution of the traffic density (the traffic load per unit area) can be approximated by the log-normal or Weibull distribution depending on time and space.

Mirahsan et al. [12] used maps of Paris, France to study the spatial traffic heterogeneity of outdoor users in dense areas of the city center. They found that the statistical distribution of spatial metrics is close to Weibull. Their results show that the building topology in a city imposes a significant degree of heterogeneity on the spatial distribution of the wireless traffic.

Taufique et al. [14] investigated the problem of planning future cellular networks. They noticed that the cell size increasingly adapts to the spatial traffic variation. Instead of having the same cell size throughout, areas with low traffic density can have larger cells compared to areas with high traffic density, resulting in energy and cost savings. As planning future cellular networks faces heterogeneous and ultra dense networks, the issue is to find the optimal base station placement jointly for macrocells and small cells in a non uniform user density scenario. They showed an example of such a deployment for a Gaussian spatial user distribution.

Wang et al. [13] characterized temporal and spatial dynamics in cellular traffic through a big cellular usage dataset covering 1.5 million users and 5,929 cell towers in a major city of China. Their results reveal highly non-uniform spatial distribution of the traffic density.

\section{CONCLUSIONS}

In this paper, we have shown that adopting inhomogeneous spatial node distribution leads to much different results on LoRaWAN capacity than that reported previously. The existing measurement studies of the traffic density in cellular networks showed high diversity of the node density in urban settings. We expect that LoRaWAN networks will follow the same deployment pattern with the placement of gateways close to high density areas.

We have used the model by Georgiou and Raza [4] to analyze the capacity of a LoRaWAN cell for various types of SF allocations: equidistant, SNR-based, and PDR-based. We can draw several conclusions from the numerical results presented in this paper:

- For a required PDR level and a target communication range, we can find an allocation of annuli $l_{j}$ that results in the maximal number of nodes that benefit from the PDR level.

- There is a natural trend towards configurations composed of smaller cells that concentrate nodes close to the gateway.
In this way, nodes benefit from low SF, which also means lower energy consumption.

- To provide the required PDR level to more nodes, we need to consider multiple gateways that will increase the overall capacity while maintaining moderate energy consumption.

In future work, we plan to explore a model in which the density of nodes is a continuous distribution in function of the distance from the gateway, which may better reflect realistic deployment scenarios.

We also want to develop models for capacity prediction in case of multiple gateways.

\section{ACKNOWLEDGMENTS}

This work has been partially supported by the French Ministry of Research project PERSYVAL-Lab under contract ANR-11-LABX0025-01.

\section{REFERENCES}

[1] LoRa ${ }^{\mathrm{TM}}$ Alliance. A Technical Overview of LoRa and LoRaWAN.

[2] A. J. Berni and W. Gregg. On the Utility of Chirp Modulation for Digital Signaling. IEEE Trans. Commun., 21, 1971.

[3] Nicolas Sornin. LoRaWAN 1.1 Specification. Technical report, LoRa Alliance, October 2017.

[4] Orestis Georgiou and Usman Raza. Low Power Wide Area Network Analysis: Can LoRa Scale? IEEE Wireless Commun. Letters, 6(2):162-165, 2017.

[5] Zhuocheng Li et al. 2D Time-Frequency Interference Modelling Using Stochastic Geometry for Performance Evaluation in Low-Power Wide-Area Networks. In 2017 ICC, May 2017.

[6] Antoine Waret et al. LoRa Throughput Analysis with Imperfect Spreading Factor Orthogonality. IEEE Wireless Communications Letters, 2018.

[7] Aamir Mahmood et al. Scalability analysis of a lora network under imperfect orthogonality. IEEE Trans. Industrial Informatics, 15(3):1425-1436, 2019.

[8] Martin Heusse et al. How Many Sensor Nodes Fit in a LoRAWAN Cell? submitted for publication, 2019.

[9] Christelle Caillouet et al. Optimal SF Allocation in LoRaWAN Considering Physical Capture and Imperfect Orthogonality. In 2019 IEEE GLOBECOM Conference, 2019.

[10] U. Gotzner and R. Rathgeber. Spatial Traffic Distribution in Cellular Networks. In VTC '98. 48th IEEE Vehicular Technology Conference. Pathway to Global Wireless Revolution (Cat. No.98CH36151), volume 3, pages 1994-1998 vol.3, May 1998.

[11] Dongheon Lee et al. Spatial Modeling of the Traffic Density in Cellular Networks. IEEE Wireless Commun., 21(1):80-88, 2014.

[12] Meisam Mirahsan et al. Measuring the Spatial Heterogeneity of Outdoor Users in Wireless Cellular Networks Based on Open Urban Maps. In 2015 ICC, pages 2834-2838, 2015

[13] Xu Wang et al. Spatio-Temporal Analysis and Prediction of Cellular Traffic in Metropolis. In 25th IEEE ICNP, pages 1-10, 2017.

[14] Azar Taufique et al. Planning Wireless Cellular Networks of Future: Outlook, Challenges and Opportunities. IEEE Access, 5:4821-4845, 2017.

[15] Semtech. SX1272/73 - $860 \mathrm{MHz}$ to $1020 \mathrm{MHz}$ Low Power Long Range Transceiver, 2017. URL https://www.semtech.com/uploads/documents/sx1272.pdf.

[16] Jetmir Haxhibeqiri et al. LoRa Scalability: A Simulation Model Based on Interference Measurements. Sensors, 17(6):1193, 2017.

[17] Claire Goursaud and Jean-Marie Gorce. Dedicated networks for IoT : PHY / MAC state of the art and challenges. EAI endorsed transactions on Internet of Things, 2015.

[18] Takwa Attia et al. Experimental Characterization of LoRaWAN Link Quality. In 2019 IEEE GLOBECOM Conference. IEEE, 2019.

[19] J. Lim and Y. Han. Spreading Factor Allocation for Massive Connectivity in LoRa Systems. IEEE Communications Letters, 22(4):800-803, April 2018.

[20] J. A. Nelder and R. Mead. A Simplex Method for Function Minimization. The Computer fournal, 7(4):308-313, 011965. 